\title{
Estudio de factibilidad del uso de sílicas mesoporosas funcionalizadas como catalizadores en destilación reactiva: esterificación de ácido acético con n-butanol
}

\author{
John A. Posada ${ }^{1}$, Oscar Giraldo ${ }^{2}$, Carlos A. Cardona ${ }^{3 \S}$ \\ ${ }^{1}$ Departamento de Ingeniería Química, Universidad Nacional de Colombia Manizales, \\ ${ }^{2}$ Departamento de Física y Quimica, Universidad Nacional de Colombia Manizales, \\ ${ }^{3 \S}$ Departamento de Ingeniería Quimica, Universidad Nacional de Colombia Manizales, \\ cardonaal@unal.edu.co
}

(Recibido:Agosto 3 de 2010 Aceptado: Mayo 5 de 2011)

\begin{abstract}
Resumen
Las silicas mesoporosas MCM-41, MCM-48, SBA-15 y HMS, funcionalizadas con el grupo propilacidosulfónico por las técnicas grafting y co-condensación, son materiales de alta estabilidad térmica y catalíticamente activos para la síntesis de acetato de n-butilo por esterificación de ácido acético con n-butanol. Estas características hacen de las silicas funcionalizadas unos catalizadores potenciales para procesos de destilación-reactiva. En este sentido se comparan económicamente el proceso tradicional de producción de acetato de n-butilo por destilación-reactiva a $0.033 \mathrm{MPa}(0.33 \mathrm{~atm})$ con el proceso a presión atmosférica $(0.101 \mathrm{MPa})$, utilizando una estrategia de optimización MINLP (Optimización de programación no lineal de mezcla entera) y el simulador comercial Aspen Plus. Los resultados mostraron que bajo condiciones óptimas el potencial económico aumenta en 9\% para el sistema a presión atmosférica.
\end{abstract}

Palabras clave: Sílica Mesoporosa, Acetato de n-Butilo, Destilación Reactiva, Optimización MINLP, Simulación de procesos.

CHEMICAL ENGINEERING

\section{Feasibility study of using funtionalized mesoporous silicas as catalysts in reactive distillation: esterification of acetic acid with $\mathbf{n}$-Butanol}

\begin{abstract}
Mesoporouses silicas MCM-41, MCM-48, SBA-15, and HMS, functionalized with the propylsulphonic acid group by grafting and co-condensation methods have high thermal stability and catalytic activity to synthesize n-butyl acetate by acetic acid esterification with n-butanol. Due to these characteristics, the mesoporouses functionalized silicas are potential catalysts for reactive distillation process. In this way, the traditional production process of n-butyl acetate by reactive distillation method, which is carried out at $0.033 \mathrm{MPa}(0.33 \mathrm{~atm})$ was compared with the process carried out at atmospheric pressure (0.101 MPa). A MINLP (Mixed-Integer Non- linear Programming Optimization) optimization strategy and the commercial simulator Aspen Plus were used. Results showed that under optimal conditions the economic potential increases $9 \%$ by the atmospheric system.
\end{abstract}

Keywords: Mesoporouses silica, n-butyl acetate, Reactive distillation, MINLP optimization, Process simulation. 


\section{Introducción}

El acetato de n-butilo es un solvente ampliamente utilizado en la industria del barniz, pintura, farmacéutica, cosmética y plásticos. Se espera que su consumo crezca debido al menor impacto ambiental comparado con solventes aromáticos y el acetato de etilo (Hanika et al. 1999, Rabindran \& Pandurangan 2005). El acetato de n-butilo se produce principalmente por esterificación de ácido acético con n-butanol. Esta reacción requiere catalizadores fuertemente ácidos como el ácido sulfúrico o p-toluensulfónico, los cuales son corrosivos y difíciles de retirar desde la mezcla reactiva. De otra parte, los catalizadores heterogéneos pueden ser removidos directamente del sistema de reacción. Diferentes materiales como resinas de intercambio iónico como Amberlyst 15, Dowex y Smopex-101 (Gelbard 2005, Blagov et al., 2006), zeolitas como la H-ZSM-5 y la zeolita-Y, óxidos sulfonatados, sólidos heteropoliácidos soportados (HPA) (Sepúlveda et al., 2005) y las sílicas mesoporosas funcionalizadas (Posada et al., 2010), permiten catalizar este tipo de sistemas reactivos. Las silicas mesoporosas son importantes debido a su alta área superficial, porosidad, diversidad de topología de poro (hexagonal, cúbica, laminar, etc.), distribución de tamaño de poro relativamente uniforme y resistencia térmica. Por esto han sido utilizadas como soporte de grupos orgánicos funcionales en la síntesis de catalizadores, especialmente para propósitos de catálisis ácida.

Las características de éstas silicas mesoporosas funcionalizadas los hacen catalizadores potenciales para sistemas de destilación reactiva, ya que uno de los principales problemas de esta tecnología es que está limitada por la resistencia térmica de catalizadores y por su reducida actividad catalítica a altas temperaturas. Este problema afecta especialmente a sistemas reactivos que requieren catalizadores con sitios ácidos de Brönsted como son las reacciones de alquilación, acilación, isomerización, eterificación, esterificación, hidratación y nitración, entre otros; debido a que los catalizadores comerciales con este tipo de sitios ácidos tienen baja estabilidad térmica. Este es el caso de la resina ácido sulfónica de intercambio ionico Amberlyst-15, que es catalíticamente activa hasta $120^{\circ} \mathrm{C}$, como fue reportado por
Gangadwala et al (2004) y por esto la producción de acetato de n-butilo por destilación reactiva debe realizarse en condiciones de vacío, debido a que la temperatura normal de ebullición de este compuesto es de $126.5^{\circ} \mathrm{C}$.

El objetivo de este articulo fue cuantificar el impacto económico que pueden tener las silicas mesoporosas funcionalizadas con grupos acidosulfónicos como catalizadores industriales de procesos que usan destilación reactiva, específicamente para el caso de producción de acetato de n-butilo. Este trabajo se soporta en los resultados reportados por Posada et al. (2010), quienes han mostrado que es posible obtener materiales con alta resistencia térmica $y$ catalíticamente activos para la reacción de esterificación de ácido acético con n-butanol. En este sentido se compara económicamente los procesos de producción de acetato de n-butilo a 0.033 y $0.101 \mathrm{MPa}$.

El diseño y la configuración de las torres de destilación se realizaron por medio del análisis de la estática, presentado detalladamente por Pisarenko et al (2001) y que permite determinar la viabilidad operacional de la torre. La simulación de los procesos se llevó a cabo por medio del simulador comercial Aspen Plus. La comparación de los dos procesos se realizó a partir de criterios energéticos y económicos, bajo condiciones de rentabilidad óptimas utilizando una estrategia de optimización de programación no lineal de mezcla entera (MINLP), la cual fue inicialmente aplicada a una torre de destilación reactiva por Ciric \& Gu (1994). Este método envuelve la minimización del costo anual sujeto a un sistema de restricciones compuesto por balances de materia, balances de energía, equilibrio de fases y expresiones cinéticas para cada etapa de la torre de destilación.

\section{Metodología.}

\subsection{Diseño y simulación de procesos}

La configuración de torre y la trayectoria tentativa de operación son determinados por medio del análisis de la estática, ampliamente explicado por Pisarenko et al. (2001). Este método permite determinar la viabilidad operacional y la mejor configuración de torre de destilación reactiva, es 
decir la localización de la zona de reacción, con una mínima información inicial y a partir del análisis de la termodinámica topológica del sistema, así como de la selección de los estados estables límites de mayor conversión, como lo muestra Serafimov et al (1999). Las suposiciones de este método llevan a realizar el análisis del sistema reactivo en estado estable bajo condiciones de equilibrio químico y operación $8 / 8$, referidas a flujos dentro de la torre y volumen de la misma. Los correspondientes parámetros de operación son la relación entre los flujos de productos destilado/alimento, volumen y localización de la zona de reacción, avance de reacción y la formulación de la separación, directa o indirecta. El catalizador utilizado para este análisis es la silica mesoporosa SBA-15 funcionalizada por el método grafting de acuerdo a lo reportado por Posada et al (2010).

Las simulaciones de las columnas de destilación reactiva se realizaron con el software comercial Aspen Plus, a partir del planteamiento termodinámico $\gamma-\varphi$, con el modelo de actividad NRTL y la ecuación de estado Hayden O'Connell, debido a la dimerización que presenta el ácido acético en la fase vapor. Estas simulaciones consideran simultáneamente los balances de materia y energía, equilibrio de fases líquidolíquido-vapor y equilibrio químico para la zona reactiva de la torre (Almeida-Rivera et al. 2004).

\subsection{Optimización MINLP}

La caracterización matemática de cada esquema de proceso requiere la definición de las variables independientes $\mathrm{y}$ dependientes, discretas $\mathrm{y}$ continuas, así como las restricciones matemáticas y operativas. Por otro lado, la rentabilidad de cada esquema de proceso es definida por una función a optimizar que considera conjuntamente los costos capitales y operativos de proceso. Para la optimización MINLP de los dos procesos, se hace un análisis en dos etapas, en primer lugar se realizan las simulaciones de destilación reactiva y entonces se calcula el potencial económico de cada proceso a partir de los resultados de la simulación. Los cálculos de los costos capitales y energéticos se hacen por medio del sistema de ecuaciones presentado en el Apéndice A.

\section{Resultados y discusión}

\subsection{Diseño y simulación de procesos}

El acetato de n-butilo se produce a partir de la reacción reversible de esterificación de ácido acético con n-butanol que se lleva a cabo en fase líquida y en la presencia de un catalizador fuertemente ácido. La caracterización termodinámica de este sistema reactivo muestra que tanto a $0.033 \mathrm{MPa}$ como a $0.101 \mathrm{MPa}$ se generan diez puntos singulares, los cuales se muestran en la Tabla 1.

Como consecuencia de esta distribución, el simplex concentracional se divide en dos regiones de destilación, mostradas en la Figura 1. Las líneas de destilación parten del azeótropo ternario de mínimo punto de ebullición n- $\mathrm{BuOH} / \mathrm{n}$ $\mathrm{BuAc} / \mathrm{H}_{2} \mathrm{O}$ y llegan, según la región de operación, bien sea al azeótropo binario $\mathrm{AcAc} / \mathrm{n}-\mathrm{BuOH}$ (Región II), o al puro n-BuAc (Región I). Así puede concluirse que existe una región de operación que permite obtener n-BuAc de alta pureza como fondo formulado. Luego de realizar el análisis de la estática, se obtiene la trayectoria tentativa de operación mostrada en la Figura 1.

Esta va desde el vértice del n-BuAc y se aproxima al vértice del $n-\mathrm{BuOH}$ por la arista que contiene a estos dos componentes, pero debido a la presencia de la separatríz cuaternaria la trayectoria continúa el acercamiento hacia el AcAc sobre esta superficie, hasta llegar finalmente al azeótropo binario $\mathrm{H}_{2} \mathrm{O} / \mathrm{n}$-BuAc. La trayectoria de operación también indica que la parte que corresponde a la sección inferior de la columna está ubicada en el espacio de reacción directa, es decir, la zona de reacción debe ubicarse en la parte inferior de la columna, mientras que la parte superior corresponde a la zona de rectificación. Adicionalmente el análisis de la estática indicó que la relación de alimentación a la torre debe ser estequiométrica. 
Tabla 1. Puntos Singulares del Sistema de Esterificación de Ácido Acético con n-Butanol

\begin{tabular}{|c|c|c|c|c|c|c|c|}
\hline $\begin{array}{c}\text { Presión } \\
\text { [Mpa] }\end{array}$ & $\begin{array}{c}\text { Temp. } \\
{\left[{ }^{\circ} \mathrm{C}\right]}\end{array}$ & Tipo & $\begin{array}{c}\text { No. } \\
\text { Comp. }\end{array}$ & AcAc & n-BuOH & n-BuAc & $\mathrm{H}_{2} \mathrm{O}$ \\
\hline 0.033 & 63.50 & Heterogéneo & 3 & 0 & 0.0672 & 0.2375 & 0.6953 \\
\hline 0.101 & 90.48 & & & & 0.1013 & 0.1978 & 0.7009 \\
\hline 0.033 & 63.72 & Heterogéneo & 2 & 0 & 0 & 0.2928 & 0.7072 \\
\hline 0.101 & 90.95 & & & & & 0.2821 & 0.7179 \\
\hline 0.033 & 66.21 & Heterogéneo & 2 & 0 & 0.2283 & 0 & 0.7717 \\
\hline 0.101 & 92.52 & & & & 0.2513 & & 0.7487 \\
\hline 0.033 & 71.63 & Homogéneo & 1 & 0 & 0 & 0 & 1 \\
\hline 0.101 & 100.00 & & & & & & \\
\hline 0.033 & 86.59 & Homogéneo & 2 & 0 & 0.5683 & 0.4317 & 0 \\
\hline 0.101 & 116.90 & & & & 0.778 & 0.222 & \\
\hline 0.033 & 89.41 & Homogéneo & 1 & 0 & 1 & 0 & 0 \\
\hline 0.101 & 117.68 & & & & & & \\
\hline 0.033 & 85.29 & Homogéneo & 1 & 1 & 0 & 0 & 0 \\
\hline 0.101 & 118.01 & & & & & & \\
\hline 0.033 & 89.98 & Homogéneo & 3 & 0.2723 & 0.1729 & 0.5548 & 0 \\
\hline 0.101 & 122.55 & & & 0.4524 & 0.336 & 0.2117 & \\
\hline 0.033 & 92.78 & Homogéneo & 2 & 0.3934 & 0.6066 & 0 & 0 \\
\hline 0.101 & 122.62 & & & 0.4876 & 0.5124 & & \\
\hline 0.033 & 91.66 & Homogéneo & 1 & 0 & 0 & 1 & 0 \\
\hline 0.101 & 126.01 & & & & & & \\
\hline
\end{tabular}


La diferencia radical entre los dos procesos evaluados está dada por las dos bombas de vacío requeridas para alcanzar la presión de operación de $0.033 \mathrm{MPa}$, el resto del esquema sigue siendo la torre de destilación reactiva y el decantador, como muestra la Figura 2.

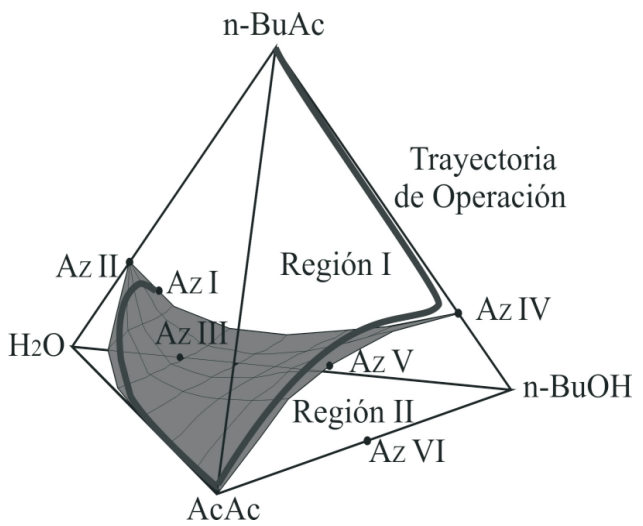

Figura 1. Mapa de curvas de residuo del sistema reactivo
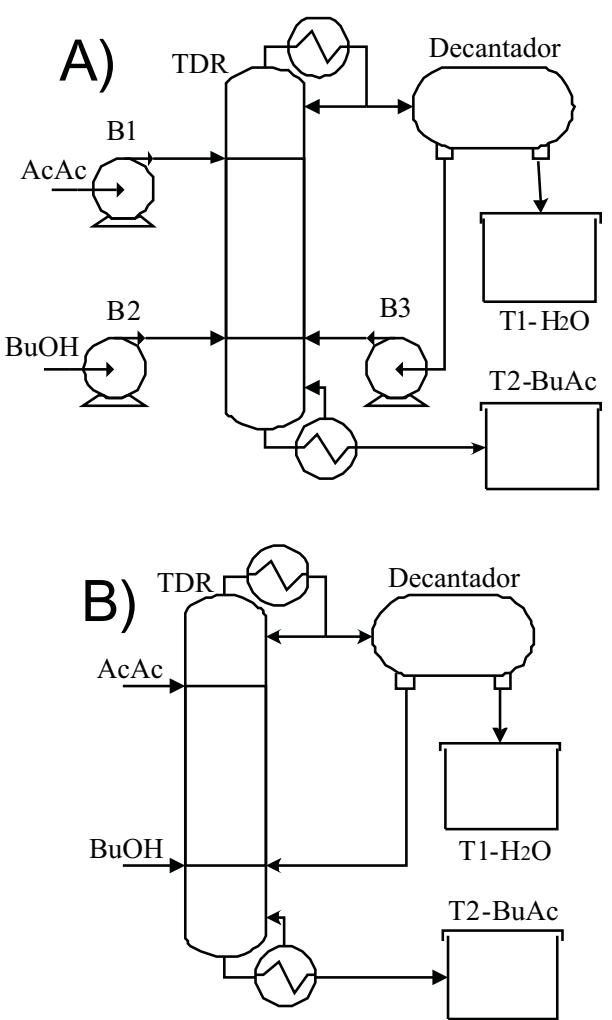

Figura 2. Esquema del Proceso de Producción de nButilacetato por destilación reactiva. A) Proceso a 0.033MPa y B) Proceso a 0.101 MPa

\subsection{Optimización de proceso}

La caracterización de las variables de proceso toma como base el trabajo realizado por Gangadwala \& Kienle (2007), como se muestra a continuación.

- Variables discretas independientes: Presión de operación (0.033 o $0.101 \mathrm{MPa}$ ); número de etapas; tipo de etapa (separación o reacción); número de corrientes de alimentación y etapas de alimentación.

- Variables continuas independientes: Relación de reflujo; relación molar de alimento y temperatura del decantador.

- Variables continuas dependientes: Volumen de la torre; volumen del rehervidor; volumen del condensador; Área de los intercambiadores de calor, Área de las bombas, volumen del decantador y volumen del eyector.

- Restricciones matemáticas: Balances de materia; balances de energía, equilibrios de fases y expresiones cinéticas.

- Restricciones operativas: Relación de alimento de reactivos (45 a $0.55 \%$ mol AcAc); pureza de productos (superior al 98\% molar) y número máximo de etapas (50 etapas).

\section{Función objetivo a optimizar}

Esta función representa el potencial económico anualizado para el proceso de producción de acetato de n-butilo por destilación reactiva, con una amortización de los costos capitales de 10 años. La ecuación planteada considera conjuntamente los costos capitales, operativos y los precios internacionales de los reactivos y del acetato de n-butilo grado uretano. Los costos capitales son dependientes de la dimensión de los equipos, por lo tanto para esta parte de la función objetivo los parámetros de diseño son: diámetro y altura de la torre, así como el área efectiva de transferencia para el condensador, rehervidor, 
eyectores y decantador. Además cada tipo de etapa en la torre (separación o reacción) requiere plantear una función específica de costos. Por otro lado los costos operativos involucran los gastos energéticos directos de las bombas de vacío, el consumo energético de los fluidos de servicio y su costo de compra. La función objetivo se expresa matemáticamente por la Eq. (1), que es complementaria al sistema de ecuaciones presentado en el Apéndice A.

$$
P E=\sum_{i=1}^{n p} F_{P_{i}} \cdot C_{P_{i}}-\sum_{i=1}^{n r} F_{R_{i}} \cdot C_{R_{i}}-\sum_{i} C_{C E q u},{ }_{i}-\sum_{i} C_{C E n e},_{i}
$$

El potencial económico se determinó en primer lugar para el proceso de síntesis realizado a presión de vacío $(0.033 \mathrm{MPa})$. Se variaron secuencialmente: la relación de reflujo (desde el reflujo mínimo hasta 20 veces su valor), el número total de etapas (5-50), el tipo de etapas (reactivas y no-reactivas), la etapa de alimentación de reactivos frescos, la etapa de alimentación de reactivos recirculados y el porcentaje de alimentación de AcAc fresco (45-55\% molar). Entonces se calcularon los costos capitales, energéticos y el potencial económico. Algunos resultados se muestran en la Figura 3.a. Para el proceso a vacio, el máximo potencial económico se alcanza para una torre con 25 etapas, donde la relación molar de reflujo es 0.8265 , con sección reactiva entre las etapas 8 y 18 ; la corriente de alimento fresco es adicionada sobre la etapa 7 y la corriente de reciclo sobre la etapa 17.

La configuración que genera el mayor potencial económico llega a un valor de 3'641.090 US\$/año, para una base de cálculo de $200 \mathrm{kgmol} / \mathrm{h}$ de mezcla fresca alimentada. Aunque valores similares se alcanzan para configuraciones con 25 etapas, modificaciones solo en la sección empacada de la torre o en la etapa de alimentación de cualquiera de las corrientes pueden generar variaciones estimadas en un $1 \%$ de la rentabilidad del proceso.
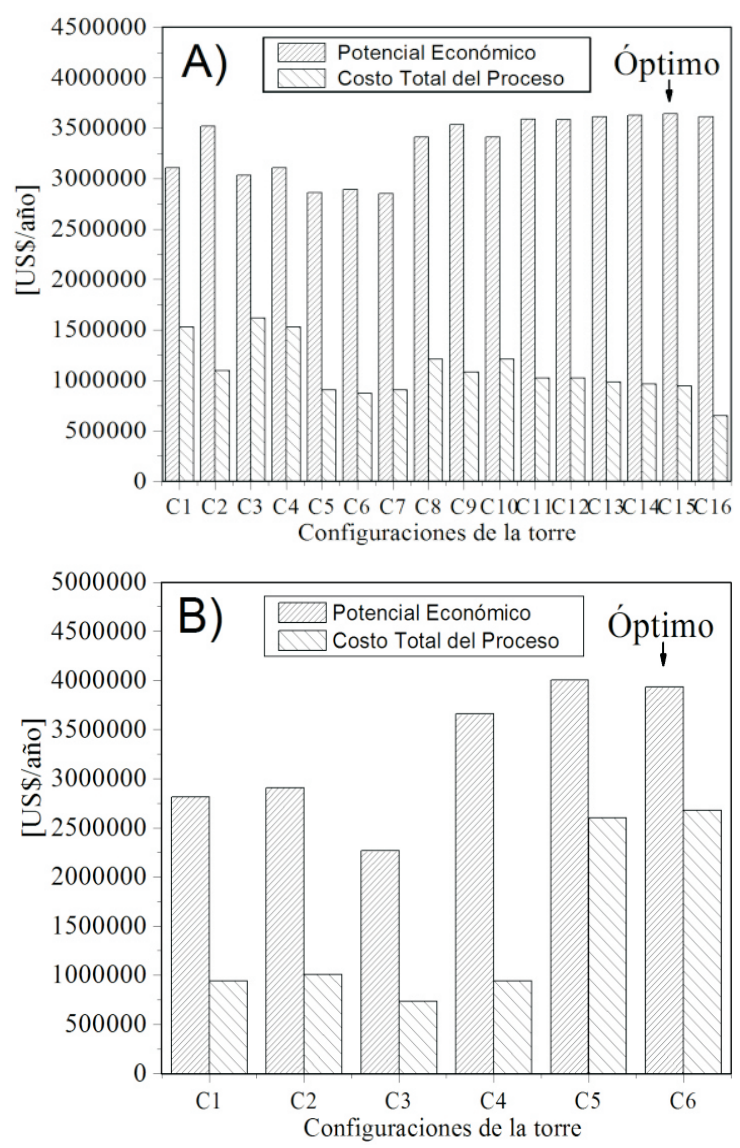

Figura 3. Diagrama de barras del potencial económico y costo total del proceso. A) 0.033 MPa y B) 0.101 MPa.

El mismo planteamiento se realizó para el proceso de síntesis a presión atmosférica. De nuevo el mayor potencial económico se obtiene para el proceso realizado con un total de 25 etapas, de las cuales 11 son reactivas y se encuentran ubicadas entre las etapas 7-17. Las corrientes de alimento fresco y de reciclo están en las etapas 6 y 16, respectivamente. Para este caso la relación molar de reflujo es 4.3469. La Figura 3.b. muestra algunos resultados del cálculo del potencial económico para el proceso a presión atmosférica. El máximo potencial económico alcanzado para este caso es de 4'001.911 US\$/año, con la misma base de cálculo utilizada para el proceso en condición de vacío. La comparación entre los dos procesos de síntesis indica que el potencial económico para el proceso realizado a presión atmosférica es $9 \%$ mayor que el proceso realizado a condición de vacío. 
Aunque la relación de reflujo es cinco veces superior para el proceso a presión atmosférica, se nota un aumento importante en su potencial económico debido a que, aun bajo estas condiciones es menor el requerimiento energético del proceso. Este fenómeno puede explicarse aún a partir de la termodinámica del sistema. La separatríz cuaternaria obtenida a $0.033 \mathrm{MPa}$ genera una región de destilación de menor tamaño que para el sistema a $0.101 \mathrm{MPa}$, lo que implica un perfil de menor volatilidad relativa al interior de la columna de destilación reactiva, llevando a un mayor requerimiento energético del re hervidor para alcanzar el grado de separación establecido en las restricciones del modelo.

Los resultados indican que aprovechar esta ventaja económica desde el punto de vista tecnológico implica remover los equipos requeridos para la generación de vacío y de pequeños cambios en la configuración de la columna, como es desplazar en una etapa la sección reactiva y las corrientes de alimentación. Finalmente se debe realizar un aumento en la relación molar de reflujo de la torre hasta 4.3469.

\section{Conclusiones}

Las silicas mesoporosas funcionalizadas con grupos acidosulfónicos, catalíticamente activas para la reacción de esterificación de ácido acético con n-butanol y su alta resistencia térmica, permiten expandir las limitaciones operativas que tiene la producción de acetato de n-butilo por destilación reactiva, desde una presión inferior a la atmosférica hasta presión atmosférica. Esta modificación en el esquema tecnológico implica una reducción en la cantidad de equipos de acondicionamiento y un cambio en el consumo energético global del sistema de procesamiento, afectando directamente la economía del proceso. Entonces, a partir de la herramienta de optimización MINLP se pudo establecer que cuando el proceso de producción de acetato de nbutilo por destilación reactiva es realizado a presión atmosférica se logra un aumento del $9 \%$ en el potencial económico respecto al proceso realizado a $0.033 \mathrm{MPa}$. La viabilidad tecnológica de los dos procesos de destilación se demostró por medio de herramientas de diseño y simulación, como el análisis de la estática y el uso del simulador comercial Aspen Plus.

\section{Nomenclatura}

$A_{\text {Con }}$ área de transferencia de calor del condensador $\left(\mathrm{m}^{2}\right)$

$A_{\text {Reh }} \quad$ área de transferencia de calor del reherbidor $\left(m^{2}\right)$

$C_{1-12} \quad$ factores de costos

$C_{B V} \quad$ costo de bombas de vacio (moneda unitaria US\$)

$C_{C a l}$ costo de calentamiento (moneda unitaria US\$ $/ \mathrm{kW}$

$C_{C C} \quad$ costo capital de la columna (moneda unitaria US\$)

$C_{C E q u, i} \quad$ costo capital del equipo i-ésimo anualizado (moneda unitaria US\$/año)

$C_{C E n e, i} \quad$ costo capital de la energía i-ésima anualizada (moneda unitaria US\$)/año

$C_{\text {Cor }} \quad$ costo de la coraza de la columna (moneda unitaria US\$)

$C_{\text {Dec }} \quad$ costo del decantador (moneda unitaria US\$)

$C_{E \mid R} \quad$ costo de etapas reactivas (moneda unitaria US\$)

$C_{E \mid S} \quad$ costo etapas de separación (no reactivas) (moneda unitaria US\$)

$C_{\text {Enf }} \quad$ costo de enfriamiento (moneda unitaria US\$ $/ \mathrm{kW}$

$C_{\text {Int }} \quad$ costo de intercambiador de calor (moneda unitaria US\$)

$C_{\text {on-AcAco }}$ concentración de ácido acético alimentado a la columna $\left(\mathrm{kmol} / \mathrm{m}^{3}\right)$

$C_{\text {оп-ВиОно }}$ concentración de n-butanol alimentado a la columna $\left(\mathrm{kmol} / \mathrm{m}^{3}\right)$

$C_{P i} \quad$ costo del producto i-ésimo (moneda unitaria US\$/mol)

$C_{R i} \quad$ costo del reactivo i-ésimo (moneda unitaria US\$ $/ \mathrm{mol}$ )

$D_{\text {Col }} \quad$ diámetro de la columna $(m)$

$D_{R L} \quad$ diámetrodel reactorlateral $(m)$

$E_{E l e} \quad$ potenciaenergía eléctricade la bomba $(\mathrm{kW})$

$F_{A c A c 0}$ flujo de ácido acético alimentado en la columna $(\mathrm{kmol} / \mathrm{s})$

$F_{C} \quad$ factor- $F\left(\mathrm{~Pa}^{0.5}\right)$, para un espacio entre platos de 12 in es $1.5\left(\mathrm{~Pa}^{0.5}\right)$

$F_{\text {Des }} \quad$ flujo de destilado $(\mathrm{kmol} / \mathrm{s})$

$F_{P i} \quad$ flujo del producto i-ésimo anualizado (kmol/año) 
$F_{P i} \quad$ flujo del reactivo i-ésimo anualizado (kmol/año)

$H^{0} \quad$ altura considerada para el rehervidor y el condensador $(m)$

$H_{\text {Col }} \quad$ alturade la columna $(m)$

HETP alturade una etapa en la columna $(m)$

$H_{R L} \quad$ alturadel reactor lateral (m)

$K_{W} \quad$ coeficiente de transferencia de calor $\left(\mathrm{kJ} / \mathrm{s} \mathrm{m}^{2}\right.$

K)

$M_{\text {Cat }} \quad$ carga total de catalizador (Amberlyst 15) en la columna (kg cat)

$M_{\text {Cat Et }} \quad$ carga máxima de catalizador (Amberlyst 15) por etapa ( $k g$ cat)

$M_{\text {Cat } R L} \quad$ masa de catalizador del reactor lateral $(\mathrm{kg}$ cat)

$M W_{i} \quad$ masa molecular del componente i-esimo $(\mathrm{kg} / \mathrm{kmol})$

$M W_{\text {MDec }}$ masa molecular de la mezcla del decantador $(\mathrm{kg} / \mathrm{kmol})$

$M \& S \quad$ indice de Marshally Swift

$N \quad$ número total de etapas

$N_{R} \quad$ número de etapas reactivas

$P \quad$ presión de operación (bar)

$P_{B V} \quad$ potenciade bombade vacio $(\mathrm{kW})$

$P E \quad$ potencial económico (moneda unitaria US\$/año)

$Q_{\text {Con }} \quad$ utilidad calórica del condensador $(\mathrm{kJ} / \mathrm{s})$

$Q_{I n t} \quad$ utilidad calórica del intercambiador de calor $(\mathrm{kJ} / \mathrm{s})$

$Q_{\text {Reh }} \quad$ utilidad calórica del rehervidor $(\mathrm{kJ} / \mathrm{s})$

$T \quad$ temperatura $(K)$

$T_{\text {Reh }} \quad$ temperatura en el rehervidor $(K)$

$\Delta T_{\text {Con }}$ gradiente de temperatura en transferencia de calor, condensador $(K)$

$\Delta T_{L M \mid n t}$ gradiente de temperatura en transferencia de calor, intercambiador $(K)$

$\Delta T_{\text {Reh }} \quad$ gradiente de temperatura para transferencia de calor en rehervidor $(K)$

$V_{\text {Dec }} \quad$ volumen del decantador $\left(\mathrm{m}^{3}\right)$

$V_{\text {Reh }} \quad$ velocidad de flujo de vapor en el rehervidor ( $\mathrm{kmol} / \mathrm{h})$

$V_{R L} \quad$ volumen del reactor lateral $\left(\mathrm{m}^{3}\right)$

Letras griegas:

$\rho_{\text {Cat }} \quad$ densidad del catalizador $\left(\mathrm{kg} / \mathrm{m}^{3}\right)$

$\tau \quad$ tiempo de residencia en el decantador $(h)$
Apéndice A: sistema de ecuaciones para el cálculo del potencial económico.

(Ver definición de variables y unidades en nomenclatura)

\section{Diámetro de la columna}

$D_{C o l}=\sqrt{\frac{V_{\mathrm{Re} h}^{4}}{\pi F_{C}} \sqrt{\frac{R T_{\mathrm{Reh}}}{P} N\left(\sum_{i=1}^{n c} M W_{i} y_{i}\right)}}$

\section{Altura de la columna}

$H_{\text {Col }}=(N-2) H E T P+H^{0}$

\section{Carga máxima de catalizador por etapa}

$M_{C a t \mid E t}=150 \frac{\pi}{4} D_{C o l}^{2} H E T P$

\section{Carga total de catalizador}

$$
M_{\text {Cat }}=M_{\text {Cat } \mid E t} N_{R}
$$

Área de rehervidor

$$
A_{\operatorname{Re} h}=\frac{Q_{\operatorname{Re} h}}{K_{W} \Delta T_{\operatorname{Re} h}}
$$

\section{Área de condensador:}

$$
A_{\text {Con }}=\frac{Q_{\text {Con }}}{K_{W} \Delta T_{\text {Con }}}
$$

\section{Costo de las etapas de separación}

$C_{E \mid S}=C_{6} D_{C o l}^{C 7} \operatorname{HETP}^{C 8}\left(N-N_{R}\right)$

\section{Costo de las etapas reactivas}

$$
C_{E \mid R}=\left(C_{9} D_{\text {Col }}^{2} H E T P\right) N_{R}
$$

\section{Costo de la coraza de la columna}

$C_{\text {Cor }}=C_{3} D_{\text {Col }}^{C 4} H_{C o l}^{C 5}$

\section{Costo de condensador y de rehervidor}

$C_{C o n \mid \operatorname{Re} h}=C_{1}\left(A_{\mathrm{Re} h}^{C 2}+A_{C o n}^{C 2}\right)$

\section{Costo capital de la columna}

$$
C_{C C}=C_{E \mid S}+C_{E \mid R}+C_{C o r}+C_{C o n \mid \operatorname{Re} h}
$$




\section{Volumen del decantador}

$$
V_{D e c}=\frac{F_{D e s} M W_{M D e c} \tau_{D e c}}{\rho_{M D e c}}
$$

\section{Costo del decantador}

$$
\begin{aligned}
& C_{\text {Dec }}=4629.3 \cdot\left(\frac{4 V_{\text {Dec }}}{3 \pi}\right)^{0.7} \cdot \\
& {\left[2.86+1.694 \cdot 2.25 \cdot\left(10.01-7.408 \ln P+1.395(\ln P)^{2}\right)\right]}
\end{aligned}
$$

\section{Área de intercambiador de calor}

$A_{\text {Int }}=\frac{Q_{\text {Int }}}{U \Delta T_{L M \mid I n t}}$

\section{Costo intercambiador de calor}

$C_{\text {Int }}=450 A_{\text {Int }}^{0.7}(1.65+1.5 \cdot 2.25)$

\section{Costo bombas de vacío}

$C_{B V}=9.381\left(\frac{M \& S}{280}\right) P_{B V}$

\section{Costo energía eléctrica}

$C_{\text {Ele }}=C_{12} E_{\text {Ele }}$

\section{Costo de enfriamiento}

$C_{E n f}=C_{11} Q_{E n f}, \quad$ (Para el Condensador)

\section{Costo de calentamiento}

$$
C_{C a l}=C_{10} Q_{\text {Cal }}, \quad \text { (Para el Rehervidor) }
$$

\section{Constantes}

$$
\begin{array}{cccc}
C_{1}=3,800.0 ; & \mathrm{C}_{2}=0.65 ; & \mathrm{C}_{3}=8200.0 ; & \mathrm{C}_{4}=0.9 ; \\
\mathrm{C}_{5}=1.0 ; & C_{6}=3,600.0 ; & \mathrm{C}_{7}=1.5 ; & \mathrm{C}_{8}=1.0 ; \\
\mathrm{C}_{9}=25,000.0 ; & \mathrm{C}_{10}=250.0 ; & C_{11}=100.0 ; & \mathrm{C}_{12}=230 ; \\
\mathrm{HEPT}=0.33 \mathrm{~m} ; & \mathrm{H}^{0}=4.0 \mathrm{~m} ; & \Delta T_{\mathrm{Re} h}=350.0 \mathrm{~K} ; & \Delta \mathrm{T}_{\mathrm{Con}}=35.0 \mathrm{~K} ; \\
\mathrm{K}_{\mathrm{W}}=8.314 \mathrm{~kJ} / \mathrm{sm}^{2} \mathrm{~K} ; & R=8.314 \mathrm{~kJ} / \mathrm{kmolK} ;
\end{array}
$$

\section{Referencias bibiográficas.}

Almeida-Rivera, C.P., Swinkels, P.L.J., \& Grievink, J. (2004). Designing reactive distillation processes: present and future. Computers and Chemical Engineering 28, 1997-2020.

Blagov, S., Parada, S., Bailer, O., Moritz, P., Lam, D., Weinand, R., \& Hassea, H. (2006). Influence of ion-exchange resin catalysts on side reactions of the esterification of n-Butanol with acetic acid. Chemical Engineering Science 61, 753-765.

Ciric, A.R., \& Gu, D. (1994).Synthesis of nonequilibrium reactive distillation processes by MINLP optimization, AIChE Journal 40, 1479-1487.

Gangadwala, J., Kienle, A., Stein, E., \& Mahajani, S. (2004).Production of Butyl Acetate by Catalytic Distillation: Process Design Studies. Industrial and Engineering Chemistry Research 43, 136-143.

Gangadwala, J., \& Kienle, A. (2007). MINLP optimization of butyl acetate synthesis. Chemical Engineering and Processing: Process Intensification 46, 107-118.

Gelbard, G. (2005). Organic Synthesis by Catalysis with Ion-Exchange Resins (Review). Industrial and Engineering Chemistry Research 44, 8468-8498

Hanika, J., Kolena, J., \& Smejkal, Q. (1999). nButyl acetate via reactive distillation modelling and experiment. Chemical Engineering Science 54, 5205-5209.

Pisarenko, Y.A., Serafimov, L.A., Cardona, C.A., Efremov, D.L., \& Shuwalov, A.S. (2001). Reactive distillation design: analysis of the process statics. Reviews in Chemical Engineering 17(4), 253-327.

Posada, J.A., Cardona, C.A., \& Giraldo, O. (2010). Comparison of acid sulfonic mesostructured silicas for 1-butylacetate synthesis. Materials Chemistry and Physics, Article in press: doi:10.1016/j.matchemphys.2010.01.027.

Rabindran, J.B., \& Pandurangan, A. (2005). A highly efficient catalyst for the esterification of acetic acid using n-butyl alcohol. Journal of Molecular Catalysis A: Chemical 237, 146-154. 
Sepúlveda, J.H., Yori, J.C., \& Vera, C.R. (2005). Repeated use of supported H3PW12O40 catalysts in the liquid phase esterification of acetic acid with Butanol. Applied Catalysis A: General 288, 18-24.

Serafimov, L.A., Zharov, V.T., \& Timofeyev, V.S. (1971). Rectification of multicomponent mixtures. I. Topological analysis of liquid vapor phase equilibrium diagrams. Acta Chimica Academiae Scientiarum Hungaricae 69(4), 383-396.

Serafimov, L.A., Pisarenko, Y.A., \& Kulov, N.N. (1999).Coupling Chemical Reaction with Distillation: Thermodynamic Analysis and Practical Applications. Chemical Engineering Science 54, 1383-1388. 